\title{
Ptychographic nanotomography at the Swiss Light Source
}

\author{
Manuel Guizar-Sicairos, ${ }^{a}$ Mirko Holler, ${ }^{a}$ Ana Diaz, ${ }^{a}$ Julio C. da Silva,,${ }^{a, b}$ Esther H. R. Tsai, ${ }^{a}$ \\ Oliver Bunk, ${ }^{a}$ Carlos Martinez-Perez,${ }^{c, d}$ Philip C. J. Donoghue, ${ }^{c}$ Charles H. Wellman, ${ }^{e}$ and \\ Andreas Menzel ${ }^{a}$ \\ ${ }^{a}$ Paul Scherrer Institut, Villigen PSI, 5232, Switzerland; \\ ${ }^{b}$ Currently at the European Synchrotron Radiation Facility, 71 Avenue des Martyrs, Grenoble \\ 38000, France; \\ ${ }^{c}$ University of Bristol, Life Sciences Building 24 Tyndall Avenue, Bristol, BS8 1TQ, United \\ Kingdom; \\ ${ }^{d}$ Universitat de València, Dr. Moliner 50, Burjassot, Valencia, E-46100, Spain; \\ ${ }^{e}$ University of Sheffield, Western Bank South Yorkshire, S10 2TN, United Kingdom
}

\begin{abstract}
Ptychography combines elements of scanning probe microscopy with coherent diffractive imaging and provides a robust high-resolution imaging technique. The extension of X-ray ptychography to 3D provides nanoscale maps with quantitative contrast of the sample complex-valued refractive index. We present here progress in reconstruction and post-processing algorithms for ptychographic nanotomography, as well as outline advances in the implementation and development of dedicated instrumentation for fast and precise 3D scanning at the Swiss Light Source. Compared to the first demonstration in 2010, such developments have allowed a dramatic improvement in resolution and measurement speed, with direct impact in the application of the technique for biology and materials science. We showcase the technique by detailing the measurement and reconstruction of a fossilized dispersed spore.
\end{abstract}

Keywords: Computed tomography, phase retrieval, ptychography, X-ray imaging, synchrotron radiation

\section{INTRODUCTION}

Ptychography is a coherent imaging scanning technique, in which the sample is illuminated by a confined illumination and scanned across the beam at points with substantial illumination overlap of neighboring positions. At each point of the scan a far-field diffraction pattern is measured, which provides the intensity of the Fourier transform of the wavefield exiting the object. Using information about the illumination overlap of neighboring positions, an iterative algorithm can be used to computationally retrieve the phase of the intensity measurements. In doing so, the complex-valued incident wave field and the object transmissivity are reconstructed. The resolution of such reconstructions can be orders of magnitude better than the size of the illumination and the scanning step. In practice, the resolution is limited by the stability and precision of the scanning stage and the maximum diffraction angle at which data can be collected with sufficient signal to noise ratio.

Ptychography finds applications in X-ray, electron and optical microscopy. A wealth of information can be encoded in a ptychogram, which can be later disentangled computationally, including the contributions of mutually incoherent coherent modes, ${ }^{1}$ separated wavelengths, or contributions arising from multiple scattering effects on thick specimens. For X-ray applications, ptychography has shown a great potential for nanoscale imaging with chemical contrast, or to be used in combination with fluorescence X-ray microscopy as complementary imaging contrast. $^{2-4}$ Beyond imaging, ptychography provides reliable reconstructions of the illumination which is used routinely for robust characterization of focused X-ray beams. ${ }^{5-8}$

The first experimental demonstration of X-ray ptychography and its extension to three dimensions, often named ptychographic X-ray computed tomography (PXCT) or shortened occasionally to ptychographic nanotomography, were both carried out at the Swiss Light Source (SLS). ${ }^{9}, 10$ PXCT provides nanoscale 3D maps

Further author information: (Send correspondence to Manuel Guizar-Sicairos)

Manuel Guizar-Sicairos: E-mail: manuel.guizar-sicairos@psi.ch, Telephone: +41 (0)56 3103409

X-Ray Nanoimaging: Instruments and Methods II, edited by Barry Lai,

Proc. of SPIE Vol. 9592, 95920A · C 2015 SPIE · CCC code:

0277-786X/15/\$18 - doi: $10.1117 / 12.2188313$

Proc. of SPIE Vol. 9592 95920A-1 
of the sample complex-valued refractive index. Its ability to reconstruct the phase of the transmissivity makes it amenable for use with high photon-energy X-rays thereby enabling studies of samples with several tens of microns in thickness.

Ptychographic nanotomography has continued to be developed at the SLS, including high-precision dedicated instrumentation, measurement strategies, and reconstruction and post-processing algorithms. Compared to its first demonstration ${ }^{10}$ such developments have already allowed an improvement of one order of magnitude in resolution and two orders of magnitude in image acquisition speed. ${ }^{11,12}$

Recent applications of PXCT at SLS include the use of the complex-valued index of refraction to quantify the mass density and water content of calcium silicate hydrates (CSH) ${ }^{13}$ which are the most abundant hydration products in ordinary Portland cement paste, to quantify the mineral distribution on human dentine, ${ }^{14}$ and for characterization of the nanoporous network of catalyst particles. ${ }^{15}$ Novel extensions of the technique have demonstrated element-specific three-dimensional imaging via resonant phase tomography ${ }^{16}$ and recovery of quantitative contrast for interior tomography by combination with a low-resolution overview. ${ }^{17}$ Ptychographic nanotomography has also been demonstrated for in situ measurements to study silk fiber hydration under varying humidity ${ }^{18}$ and quantify the densification and fracture of a microcomposite undercompression. ${ }^{19}$ Such experiments highlight some of the most promising characteristics of the technique by exploiting the nanoscale resolution, quantitative contrast and non-destructive character.

Here we present an application example of PXCT to image a cryptospore fossil, highlighting the measurement procedure and reconstruction techniques.

\section{SAMPLE PREPARATION AND MEASUREMENT}

A Late Ordovician early land plant spore, cryptospore tetrad, was scanned using ptychographic nanotomography. The fossilized dispersed spore was composed of sporopollenin, which was a C-O-H macromolecule, and was obtained by standard palynological acid maceration techniques using hydrochloric and hydrofluoric acids from the GHABA-1 borehole from northern Oman at a depth of 1573 meters. This borehole penetrates a thick Ordovician-Silurian sequence, consisting primarily of terrestrial and marginal marine deposits. The sample was of Late Ordovician, Caradoc age, approximately 450 millions years ago. These dispersed spores represent some of the earliest evidence of land plants on Earth.

X-ray measurements were carried out at the cSAXS beamline (X12SA), Swiss Light Source, Paul Scherrer Institut, Switzerland. The photon energy was selected to $8.7 \mathrm{keV}$ with $0.02 \%$ bandwidth by a double crystal $\mathrm{Si}(111)$ monochromator. A spatially confined illumination was obtained using a 120 micron diameter Fresnel zone plate lens with $60 \mathrm{~nm}$ outermost zone width, manufactured at the Laboratory for Micro- and Nanotechnology, Paul Scherrer Institut, Switzerland. Transverse coherence of the light incident on the zone plate was ensured by a slit, with a horizontal opening of 25 microns, located at 12.1 meters downstream from the undulator and 22 meters upstream of the Fresnel zone plate.

The specimen was glued to the top of specially designed copper sample pin. Ptychographic scans were performed on an interferometrically controlled high precision $3 \mathrm{D}$ scanning stage $\mathrm{e}^{11,20}$ with a field of view of $50 \times 18 \mu \mathrm{m}^{2}$. At 7.389 meters downstream of the sample, an Eiger photon-counting detector ${ }^{21,22}$ was used to acquire the far-field intensity patterns at each of the resulting 227 scanning points with an exposure time of 0.1 seconds. The scanning pattern followed concentric circles with a radial step size of 1.8 microns to avoid the raster-grid pathology which can introduce artifacts for regular Cartesian scanning patterns. ${ }^{23}$ The same ptychography scan was repeated at 360 angular orientations of the sample equally spaced in the range between 0 and 179.5 degrees.

\section{RECONSTRUCTION AND PROCESSING}

The ptychography reconstructions were carried out by using $300 \times 300$ pixels of the detector with the differencemap algorithm ${ }^{24}$ followed by a maximum likelihood refinement, ${ }^{25}$ resulting in a reconstruction pixel size of $46.8 \mathrm{~nm}$. These two-dimensional projections were further processed following an in-house developed procedure, ${ }^{26}$ which is briefly described in the following for completeness. First, a constant and linear phase are removed using 
the air measured around the sample as a reference. Subsequently, a region of the sample with a small number of phase residues is chosen for unambiguous unwrapping. ${ }^{27}$ The unwrapped region is used for the vertical alignment of projections by a method that extracts the vertical mass fluctuations, i.e. along the axis of rotation, and uses this function that is invariant to sample rotation to iteratively bring all projections into alignment with sub-pixel precision. The horizontal alignment is initially estimated using the horizontal center of mass of the sample and is followed by a refinement using an iterative approach using tomographic consistency. ${ }^{17,28}$ In the latter step a sinogram is low-pass filtered, a low-resolution tomographic slice is computed using filtered back projection (FBP) and physical constraints on the density values can be then applied. From the latter tomographic slice a synthetic sinogram is computed again. Horizontal alignment values are then obtained by minimizing the mean squared difference between the later computed sinogram and the original one. The method hinges on the fact that misaligned sinograms are not consistent with a three-dimensional representation. Finally, the tomogram is computed using a modified technique that allows tomography from wrapped-phase projections. ${ }^{26}$

Although the sample position is known to a precision approximately 10 nanometers for any individual twodimensional projection, ${ }^{11,20}$ upon rotation, knowledge of the relative position of the sample is limited to how close the reference interferometry mirrors are to their nominal planar and spherical shapes, which in this case have a shape error around 100 nanometers. This makes post-processing alignment of projections necessary for now to reach resolutions below 20 nanometers. However, with a stabilized temperature environment it is feasible that the surface of the mirror can be accurately characterized and that the need for post-processing alignment steps may be reduced.

The methods outlined above use all data available and all natural contrast features for sub-pixel alignment of the projections. Furthermore, they exploit consistency properties that must be satisfied for a three-dimensional object. This makes these methods very robust and amenable to automation as there is no need for user interaction to select alignment markers.

\section{RESULTS AND DISCUSSION}

As an overview of the cryptospore fossil internal structure, Fig. 1 shows sagittal and coronal tomographic sections of the specimen, each section separated by 4.68 microns. In these sections it is possible to see a faint differentiation in electron density between the specimen and the mounting glue. Although the fossil is radiation tolerant, the epoxy suffers some radiation damage, causing a small drift throughout the measurement. This mainly results in the appearance of subtle streak artifacts that become slightly more apparent in Fig. 2, which shows two axial sections perpendicular to the axis of rotation with a 2.34 micron separation. Overview three-dimensional renderings shown in Fig. 3 give a general representation of the external three-dimensional shape and differentiates the glue in a darker shade of red.

The tomographic reconstruction allows us to identify different morphological features difficult to see from more traditional techniques such as scanning electron microscopy (SEM) or transmission electron microscopy (TEM). The data shows an unfused tetrad in which walls are entirely homogeneous with no internal differentiation, lacking an outer envelope.

Ptychographic nanotomography offers the possibility of obtaining images of internal structure of microscopic samples without destroying the sample, making it amenable to posterior correlative imaging with other methods. Additionally, it avoids the possibility of introducing artifacts during cutting or milling necessary for other destructive techniques. Although it has a native electron density contrast, by measuring at photon energies corresponding to an atomic species of interest, it is possible to obtain element-specific quantitative maps through resonant phase nanotomography. ${ }^{16}$ Beyond the paleontology example shown here, ptychographic nanotomography offers great potential for nanoscale imaging in materials and biological sciences.

\section{ACKNOWLEDGMENTS}

The X-ray measurements were carried out at the cSAXS beamline (X12SA), Swiss Light Source, Paul Scherrer Institut, Switzerland. The authors are grateful to Xavier Donath for the excellent technical support. Esther Tsai is supported by SNSF grant no. 200021_152554. 

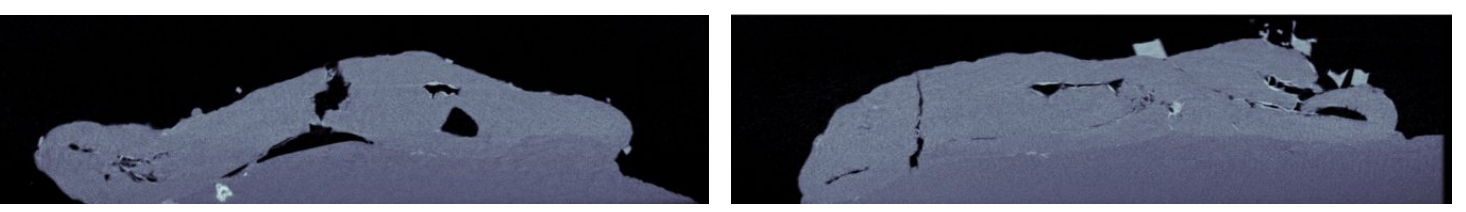

$\mathrm{e} / \AA^{3}$
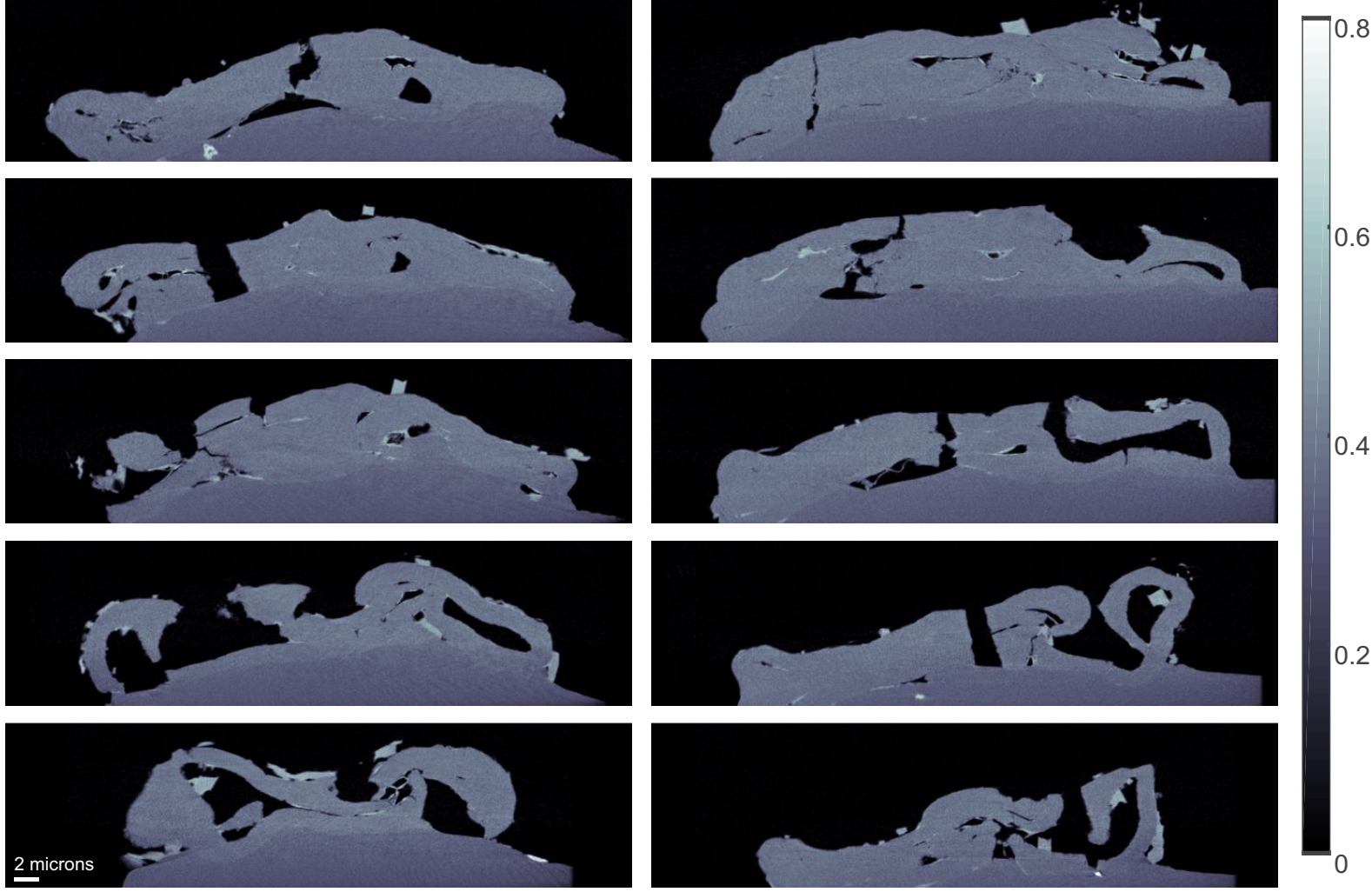

Figure 1. (left) Coronal and (right) sagittal tomographic sections of the tetrad cryptospore. Sections are spaced by 4.68 microns. The color bar and the scale bar apply to all images.
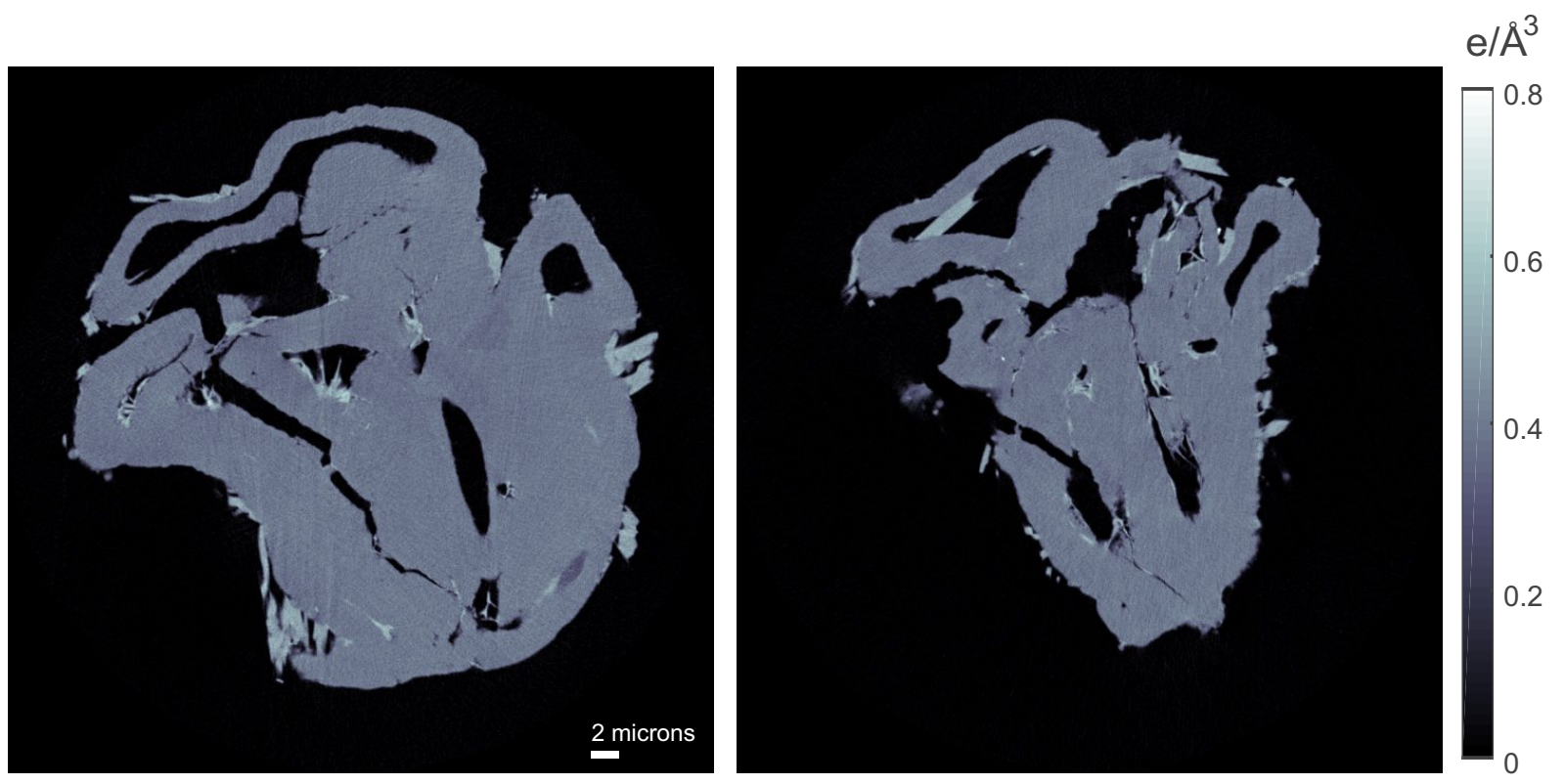

Figure 2. Two axial tomographic sections of the tetrad cryptospore. Sections are spaced by 2.34 microns. The color bar and the scale bar apply to all images. 

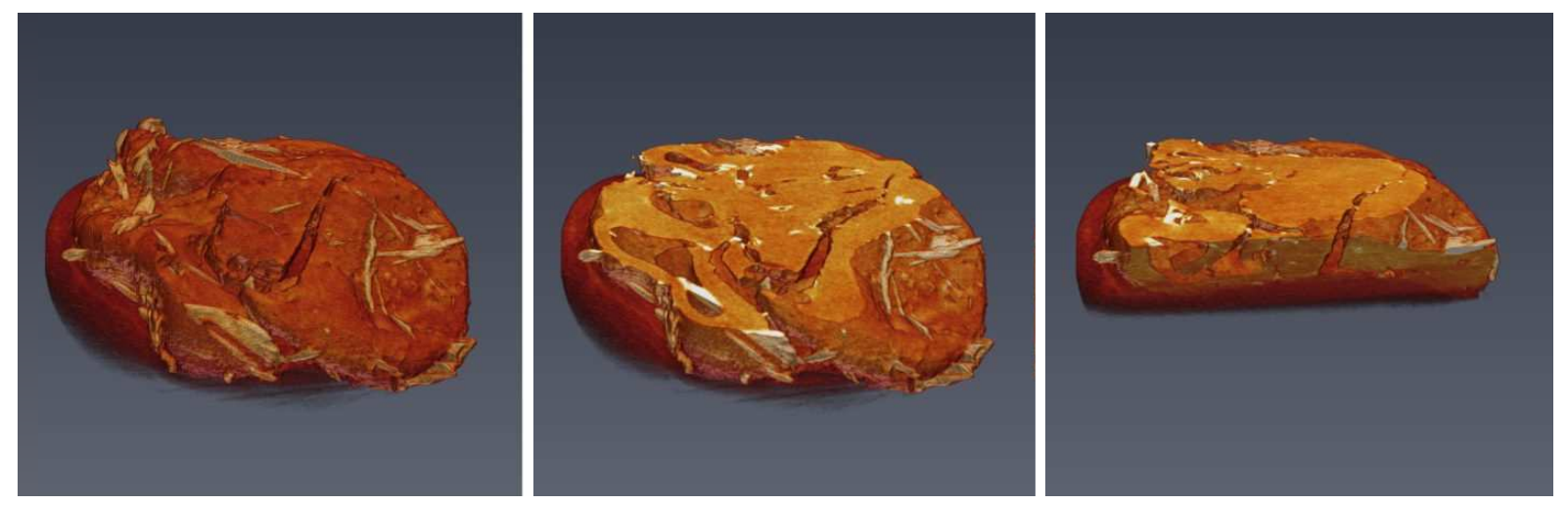

Figure 3. Three-dimensional rendering of the tetrad cryptospore.

\section{REFERENCES}

[1] Thibault, P. and Menzel, A., "Reconstructing state mixtures from diffraction measurements," Nature 494, 68-71 (2013).

[2] Vine, D. J., Pelliccia, D., Holzner, C., Baines, S. B., Berry, A., McNulty, I., Vogt, S., Peele, A. G., and Nugent, K. A., "Simultaneous x-ray fluorescence and ptychographic microscopy of cyclotella meneghiniana," Opt. Express 20, 18287-18296 (2012).

[3] Deng, J., Vine, D. J., Chen, S., Nashed, Y. S. G., Jin, Q., Phillips, N. W., Peterka, T., Ross, R., Vogt, S., and Jacobsen, C. J., "Simultaneous cryo x-ray ptychographic and fluorescence microscopy of green algae," Proc. Natl. Acad. Sci. 112, 2314-2319 (2015).

[4] Shapiro, D. A., Yu, Y.-S., Tyliszczak, T., Cabana, J., Celestre, R., Chao, W., Kaznatcheev, K., Kilcoyne, A. L. D., Maia, F., Marchesini, S., Meng, Y. S., Warwick, T., Yang, L. L., and Padmore, H. A., "Chemical composition mapping with nanometre resolution by soft x-ray microscopy," Nat. Photonics 8, 765-769 (2014).

[5] Guizar-Sicairos, M. and Fienup, J. R., "Measurement of coherent x-ray focused beams by phase retrieval with transverse translation diversity," Opt. Express 17(4), 2670-2685 (2009).

[6] Schropp, A., Boye, P., Feldkamp, J. M., Hoppe, R., Patommel, J., Samberg, D., Stephan, S., Giewekemeyer, K., Wilke, R. N., Salditt, T., Gulden, J., Mancuso, A. P., Vartanyants, I. A., Weckert, E., Schder, S., Burghammer, M., and Schroer, C. G., "Hard x-ray nanobeam characterization by coherent diffraction microscopy," Appl. Phys. Lett. 96, 091102 (2010).

[7] Guizar-Sicairos, M., Narayanan, S., Stein, A., Metzler, M., Sandy, A. R., Fienup, J. R., and Evans-Lutterodt, K., "Measurement of hard x-ray lens wavefront aberrations using phase retrieval," Appl. Phys. Lett. 98, 111108 (2011).

[8] Vila-Comamala, J., Diaz, A., Guizar-Sicairos, M., Mantion, A., Kewish, C. M., Menzel, A., Bunk, O., and David, C., "Characterization of high-resolution diffractive x-ray optics by ptychographic coherent diffractive imaging," Opt. Express 19, 21333-21344 (2011).

[9] Rodenburg, J. M., Hurst, A. C., Cullis, A. G., Dobson, B. R., Pfeiffer, F., , Bunk, O., David, C., Jefimovs, K., and Johnson, I., "Hard-X-Ray lensless imaging of extended objects," Phys. Rev. Lett. 98, 034801 (2007).

[10] Dierolf, M., Menzel, A., Thibault, P., Schneider, P., Kewish, C. M., Wepf, R., Bunk, O., and Pfeiffer, F., "Ptychographic X-ray computed tomography at the nanoscale," Nature 467, 436-439 (2010).

[11] Holler, M., Diaz, A., Guizar-Sicairos, M., Karvinen, P., Färm, E., Härkönen, E., Ritala, M., Menzel, A., Raabe, J., and Bunk, O., "Hard x-ray ptychographic computed tomography at $16 \mathrm{~nm}$ 3D resolution," Sci. Rep. 4, 3857 (2014).

[12] Thibault, P., Guizar-Sicairos, M., and Menzel, A., "Coherent imaging at the diffraction limit," J. Synchrotron Radiat. 21, 1011-1018 (2014). 
[13] da Silva, J. C., Trtik, P., Diaz, A., Holler, M., Guizar-Sicairos, M., Raabe, J., Bunk, O., and Menzel, A., "Mass density and water content of saturated never-dried calcium silicate hydrates," Langmuir 31, 3779-3783 (2015).

[14] Zanette, I., Enders, B., Dierolf, M., Thibault, P., Gradl, R., Diaz, A., Guizar-Sicairos, M., Menzel, A., Pfeiffer, F., and Zaslansky, P., "Ptychographic x-ray nanotomography quantifies mineral distributions in human dentine," Sci. Rep. 5, 9210 (2015).

[15] da Silva, J. C., Mader, K., Holler, M., Haberthr, D., Diaz, A., Guizar-Sicairos, M., Cheng, W.-C., Shu, Y., Raabe, J., Menzel, A., and van Bokhoven, J. A., "Assessment of the 3D pore structure and individual components of preshaped catalyst bodies by x-ray imaging," ChemCatChem 7, 413-416 (2015).

[16] Donnelly, C., Guizar-Sicairos, M., Scagnoli, V., Holler, M., Huthwelker, T., Menzel, A., Vartiainen, I., Müller, E., Kirk, E., Gliga, S., Raabe, J., and Heyderman, L. J., "Element-specific x-ray phase tomography of 3D structures at the nanoscale," Phys. Rev. Lett. 114, 115501 (2015).

[17] Guizar-Sicairos, M., Boon, J. J., Mader, K., Diaz, A., Menzel, A., and Bunk, O., "Quantitative interior x-ray nanotomography by a hybrid imaging technique," Optica 2, 259-266 (2015).

[18] Esmaeili, M., Fløystad, J. B., Diaz, A., Høydalsvik, K., Guizar-Sicairos, M., Andreasen, J. W., and Breiby, D. W., "Ptychographic X-ray tomography of silk fiber hydration," Macromolecules 46, 434-439 (2013).

[19] Bø Fløystad, J., Skjønsfjell, E. T. B., Guizar-Sicairos, M., Høydalsvik, K., He, J., Andreasen, J. W., Zhang, Z., and Breiby, D. W., "Quantitative 3D x-ray imaging of densification, delamination and fracture in a micro-composite under compression," Adv. Eng. Mater. 17, 545-553 (2015).

[20] Holler, M., Raabe, J., Diaz, A., Guizar-Sicairos, M., Quitmann, C., Menzel, A., and Bunk, O., "An instrument for 3D X-ray nano-imaging," Rev. Sci. Instrum. 83, 073703 (2012).

[21] Johnson, I., Bergamaschi, A., Billich, H., Cartier, S., Dinapoli, R., Greiffenberg, D., Guizar-Sicairos, M., Henrich, B., Jungmann, J., Mezza, D., Mozzanica, A., Schmitt, B., Shi, X., and Tinti, G., "Eiger: a single-photon counting x-ray detector," J. Instrum. 9, C05032 (2014).

[22] Guizar-Sicairos, M., Johnson, I., Diaz, A., Holler, M., Karvinen, P., Stadler, H.-C., Dinapoli, R., Bunk, O., and Menzel, A., "High-throughput ptychography using Eiger: scanning x-ray nano-imaging of extended regions," Opt. Express 22, 14859-14870 (2014).

[23] Dierolf, M., Thibault, P., Menzel, A., Kewish, C. M., Jefimovs, K., Schlichting, I., von Knig, K., Bunk, O., and Pfeiffer, F., "Ptychographic coherent diffractive imaging of weakly scattering specimens," New J. Phys. 12, 035017 (2010).

[24] Thibault, P., Dierolf, M., Bunk, O., Menzel, A., and Pfeiffer, F., "Probe retrieval in ptychographic coherent diffractive imaging," Ultramicroscopy 109, 338-343 (2009).

[25] Thibault, P. and Guizar-Sicairos, M., "Maximum-likelihood refinement for coherent diffractive imaging," New J. Phys. 14, 063004 (2012).

[26] Guizar-Sicairos, M., Diaz, A., Holler, M., Lucas, M. S., Menzel, A., Wepf, R. A., and Bunk, O., "Phase tomography from X-ray coherent diffractive imaging projections," Opt. Express 19, 21345-21357 (2011).

[27] Goldstein, R. M., Zebker, H. A., and Werner, C. L., "Satellite radar interferometry: Two-dimensional phase unwrapping," Radio Sci. 23, 713-720 (1988).

[28] Guizar-Sicairos, M., Boon, J. J., Mader, K., Diaz, A., Menzel, A., and Bunk, O., "Quantitative interior x-ray nanotomography by a hybrid imaging technique: Supplementary material," Optica 2 (2015). 